\title{
The benefits and risks of pembrolizumab
} in combination with chemotherapy as firstline therapy in small-cell lung cancer: a singlearm meta-analysis of noncomparative clinical studies and randomized control trials

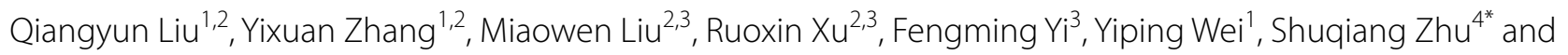
Wenxiong Zhang ${ }^{1^{*}}$ (D)

\begin{abstract}
Background: Although pembrolizumab has shown clinical benefit in patients with small-cell lung cancer (SCLC), its actual efficacy in combination with a conventional chemotherapy drug has not been determined. We performed this study to discern the efficacy and risk of pembrolizumab in combination with chemotherapy as first-line therapy in SCLC patients.

Methods: We systematically searched the PubMed, ScienceDirect, Cochrane Library, Scopus, Ovid MEDLINE, Embase, Web of Science, and Google Scholar databases for relevant studies. The main outcomes were overall survival (OS) and progression-free survival (PFS).

Results: We identified 2980 articles and included 6 studies ( 5 were noncomparative open-label studies and 1 was a randomized controlled trial [RCT]) involving 396 patients in our meta-analysis. The pooled median OS (mOS) was 9.6 months ( $95 \% \mathrm{Cl}, 8.0-11.2$ ), and the pooled median PFS (mPFS) was 4.2 months ( $95 \% \mathrm{Cl}, 2.2-6.1$ ). The 1-year overall survival rate (OSR-1y) and 6-month progression-free survival rate (PFSR-6m) were $45.1 \%(95 \% \mathrm{Cl}, 33-57.2 \%)$ and $41.6 \%$ (95\% Cl, 24.3-59\%), respectively. The objective response rate (ORR) was 38.8\% (95\% Cl, 11.9-65.67\%), disease control rate (DCR) was 69.30\% (95\% Cl, 51.6-87.0\%), complete response (CR) was 2.20\% (95\% Cl, 0.8-3.7\%), partial response (PR) was 34.70\% (95\% Cl, 7.8-61.5\%), and stable disease (SD) was 20.90\% (95\% Cl, 9.1-32.6\%). The grade 3-4 adverse effect (AE) rate was $20.88 \%(95 \% \mathrm{Cl}, 1.22-54.85 \%)$. The most common AEs were neutropenia $(90.16 \%)$, anemia (53.21\%), dysphagia (41.96\%), platelet count decrease (34.87\%), and esophagitis (32.89\%); severe AEs included neutropenia, respiratory failure, pneumonitis, acute coronary syndrome, and colitis/intestinal ischemia.
\end{abstract}

\footnotetext{
*Correspondence: zsq276446377@163.com; zwx123dr@126.com

${ }^{1}$ Department of Thoracic Surgery, The Second Affiliated Hospital of Nanchang University, 1 Minde Road, Nanchang 330006, China

${ }^{4}$ Department of Cardiovascular Surgery, The Second Affiliated Hospital of Nanchang University, Nanchang 330006, China

Full list of author information is available at the end of the article
}

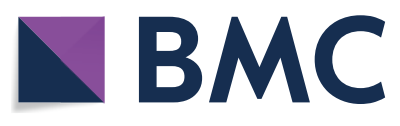

(c) The Author(s) 2021. Open Access This article is licensed under a Creative Commons Attribution 4.0 International License, which permits use, sharing, adaptation, distribution and reproduction in any medium or format, as long as you give appropriate credit to the original author(s) and the source, provide a link to the Creative Commons licence, and indicate if changes were made. The images or other third party material in this article are included in the article's Creative Commons licence, unless indicated otherwise in a credit line to the material. If material is not included in the article's Creative Commons licence and your intended use is not permitted by statutory regulation or exceeds the permitted use, you will need to obtain permission directly from the copyright holder. To view a copy of this licence, visit http://creativecommons.org/licenses/by/4.0/. The Creative Commons Public Domain Dedication waiver (http://creativeco mmons.org/publicdomain/zero/1.0/) applies to the data made available in this article, unless otherwise stated in a credit line to the data. 
Conclusions: The combination of pembrolizumab with conventional chemotherapy is an effective therapeutic schedule with acceptable and manageable efficacy and toxicity in patients with SCLC. More high-quality and welldesigned RCTs with large sample sizes are warranted to further validate our findings.

Keywords: Pembrolizumab, Chemotherapy, Small-cell lung cancer, Meta-analysis, Systematic review

\section{Novelty and impact statements}

This meta-analysis offers the most comprehensive and latest evidence for the management of small-cell lung cancer. The results suggested that pembrolizumab with conventional chemotherapy is a beneficial therapeutic schedule with assessable PFS, OS, ORR, and grade 3-4 AEs. Subgroup analyses indicated that patient age ( $<65$ years old) and SCLC phase (LS-SCLC) are the main factors affecting efficacy of pembrolizumab plus chemotherapy.

\section{Background}

As a type of invasive tumor with few treatment options and undesirable prognosis [1], small-cell lung cancer (SCLC) makes up a relatively significant proportion (18-20\%) of all new lung cancer cases, $80-85 \%$ of which are diagnosed with extensive-stage disease worldwide [2]. Platinum-based chemotherapy represents the foundation of treatment for patients with SCLC, but not much progress has been made in related therapeutic drugs [3]. Although SCLC is initially sensitive to chemotherapy and radiation, it always relapses within 6 months with a low 5-year survival rate (5\%) [4-6], and the emergence of drug resistance is still a considerable clinical challenge [7]. In recent years, immunotherapy has been recommended for the treatment of SCLC and has shown relatively good efficacy and safety.

As one of the earliest researched and developed immunotherapy drugs, pembrolizumab was approved for treating SCLC in 2019 [8]; moreover, the efficacy of pembrolizumab plus chemotherapy in NSCLC was also demonstrated that substantially improved OS and PFS [9]; however, the efficacy and risk of pembrolizumab plus conventional chemotherapy have yet to be determined [10, 11]. Charles et al. [12] demonstrated that the combination of pembrolizumab and a chemotherapy drug had better efficacy than the chemotherapy drug alone (hazard ratio [HR] of progression-free survival (PFS): 0.80 ; 95\% CI, 0.64-0.98; $p=0.0164$ ). Multiple noncomparative studies [13-17] have confirmed its acceptable efficacy with a median overall survival (mOS) of 9.7-10.8 months. To better guide clinical treatment, we performed this study to comprehensively analyze the benefit and risk of pembrolizumab plus chemotherapy.

\section{Materials and methods}

We conducted this meta-analysis following the Preferred Reporting Items for Systematic Review and Meta-Analysis (PRISMA) guidelines (Table S1) (PROSPERO registration: CRD42020218612).

\section{Search strategy}

We retrieved relevant literature through searches in PubMed, Web of Science, The Cochrane Library, ScienceDirect, Scopus, Ovid MEDLINE, and Embase. The last search was on December 20, 2020. The combined search term with Medical Subject Headings (MeSH) terms applied were "Pembrolizumab," "Chemotherapy," and "Small-cell lung cancer" (detailed search strategy in Table S2). The unpublished papers during the study and references of included studies were also searched.

\section{Selection criteria}

Articles meeting the following conditions were included:

(1) Participants: Adult patients diagnosed with SCLC, regardless of extensive-stage small-cell lung cancer (ES-SCLC) or limited small-cell lung cancer (LSSCLC) without restriction and obvious differences in sex, race, region, or nationality

(2) Interventions: Treatment arm included pembrolizumab and a chemotherapy drug whether or not a comparison had been implemented

(3) Outcomes: The primary study outcomes were OS, PFS, objective response rate (ORR), disease control rate (DCR), and grade 3-4 treatment-related adverse effect rate (grade 3-4 AEs\%). All treatmentrelated AE ranks were evaluated by the Lung Cancer Symptom Scale and Life-5 Dimension questionnaire [18]

(4) Study design: Prospective open-label clinical study and randomized control trials (RCTs)

The latest studies from 2017 to 2020 were adopted to extract data of survival outcomes, drug response, and adverse effects (AEs). We excluded meta-analyses, reviews without available original data, human experiments without pembrolizumab + chemotherapy 
exposure and relevant outcome data, animal experiments, and studies with duplicated data or only abstracts.

\section{Data extraction}

The following data were extracted by two investigators independently: first author, year of publication, registered number, country, number of participants, participant characteristics (age, sex, phase of SCLC, and Eastern Cooperative Oncology Group [ECOG] status), chemotherapy drug, pembrolizumab arm, and study design. We extracted the total relevant data and subgroup characteristics from noncomparative studies and extracted valuable data on therapeutic regimens and control groups from RCTs. Antitumor efficacy indices (survival outcomes including mPFS, mOS, OSR, and PFSR; drug responses including ORR, DCR, complete response [CR], partial response $[\mathrm{PR}]$, stable disease $[\mathrm{SD}]$, median duration of response [mDOR], and ongoing response) and AEs (total AEs and grade 3-4 AEs) were also extracted. Another investigator resolved any differences, and all original data were recorded in corresponding tables.

\section{Quality assessment}

RCTs were assessed using the Cochrane risk of bias tool and did not identify existing allocation concealment and blinding methods, as well as generated random sequences, provided complete outcome data, and reported no selective outcome without other bias [19]. GRADE quality assessment was also conducted through therapeutic regimens for the survival outcomes, drug response, and AEs to confirm the high quality of the results [20]. The noncomparative studies we included came from top journals or excellent scientists, with high quality and credibility.

\section{Statistical analysis}

We analyzed the survival outcomes and drug response of pembrolizumab combined with a conventional chemotherapy drug. We input total therapeutic regimens for the percentage of survival outcomes and number of participants and calculated the standard errors of these quasinormal distribution "rates" using Stata (version 16.0). We used the "rate" and standard errors to obtain the $95 \%$ confidence interval (CI) [21, 22]. Finally, the pooled effect size (ES), which represented the median "rate" with 95\% CI, was output [23], and we used the double arcsine method to converse and correct the rate less than 0.2 [24]. We estimated the mean using the values of the median OS and PFS, low and high end of the range, and the sample size to output the pooled ES [25]. The pooled ES was used to estimate the efficacy and risk of pembrolizumab plus chemotherapy. The heterogeneity of the included studies was assessed by the Cochrane Q chi-square test and the $I^{2}$ statistic, with $25-50 \%, 50-75 \%$, and $>75 \%$ representing low, moderate, and high heterogeneity, respectively [26]. $P<0.1$ for the $Q$ test was deemed to indicate high heterogeneity. Random-effects models were applied to all pooled ESs because the included studies were mostly single-arm (noncomparative) experiments without control groups and had a tendency toward high heterogeneity [27]. $P<0.05$ was regarded as statistically significant.

We also performed subgroup analyses of the study design (noncomparative open-label study, randomized control study), chemotherapy drug (platinum plus etoposide; platinum plus etoposide and paclitaxel), clinical setting, SCLC phase (ES-SCLC, LS-SCLC), study phase (I, II, III, I/II), median participant age ( $\leq 65$ years old, > 65 years old), region (America, Korea), and whether the treatment was combined with radiation (Yes, No).

We assessed the publication of the primary outcomes through the Egger's and Begg's linear regression tests [28]. We used Stata 16.0 (StataCorp, USA, http://www. stata.com) to conduct all statistical analyses.

\section{Results}

Search results and study quality assessment

We initially identified 2980 studies. Finally, 6 studies involving 396 patients were included in our study (Fig. 1) [12-17]. Of the 6 studies, 1 was a randomized controlled trial (RCT) [11] and 5 were noncomparative prospective open-label clinical studies [12-16]. Welsh et al. [15] and Heymach et al. [17] came from the same clinical study but with different participants (38 ES-SCLC patients and 40 LS-SCLC patients, respectively) not involving duplication.

The RCT [12] was of high quality (scored 5 points), and the noncomparative studies [13-17] we included came from top journals or excellent researchers or scholars, which guaranteed their high quality and reliability. The baseline characteristics and primary evaluating indicators of the included studies are summarized in Tables 1 and 2. According to the GRADE method, all of the results were of medium-high quality (Table S3).

\section{Antitumor efficacy}

Some studies reported mOS and its range [12, 14-17] involving 377 patients; the pooled mOS was 9.6 months (95\% CI, 8.0-11.2, $I^{2}=43.1 \%$ ) (Table S4, Fig. 2A), with the 6-month overall survival rate (OSR-6m), OSR-9m, OSR$12 \mathrm{~m}$, OSR $-15 \mathrm{~m}$, and OSR-18m being $78.50 \%$ (95\% CI, 68.9-88.1\%), 59.90\% (95\% CI, 48.2-71.6\%), 45.10\% (95\% CI, 33.0-57.2\%), 38.50\% (95\% CI, 21.8-55.3\%), and 32.20\% (95\% CI, 8.4-56.0\%) (Fig. 3), respectively.

Some studies reported mPFS and its range [12-17] involving 396 patients; the pooled mPFS was 4.2 months 
PRISMA 2009 Flow Diagram

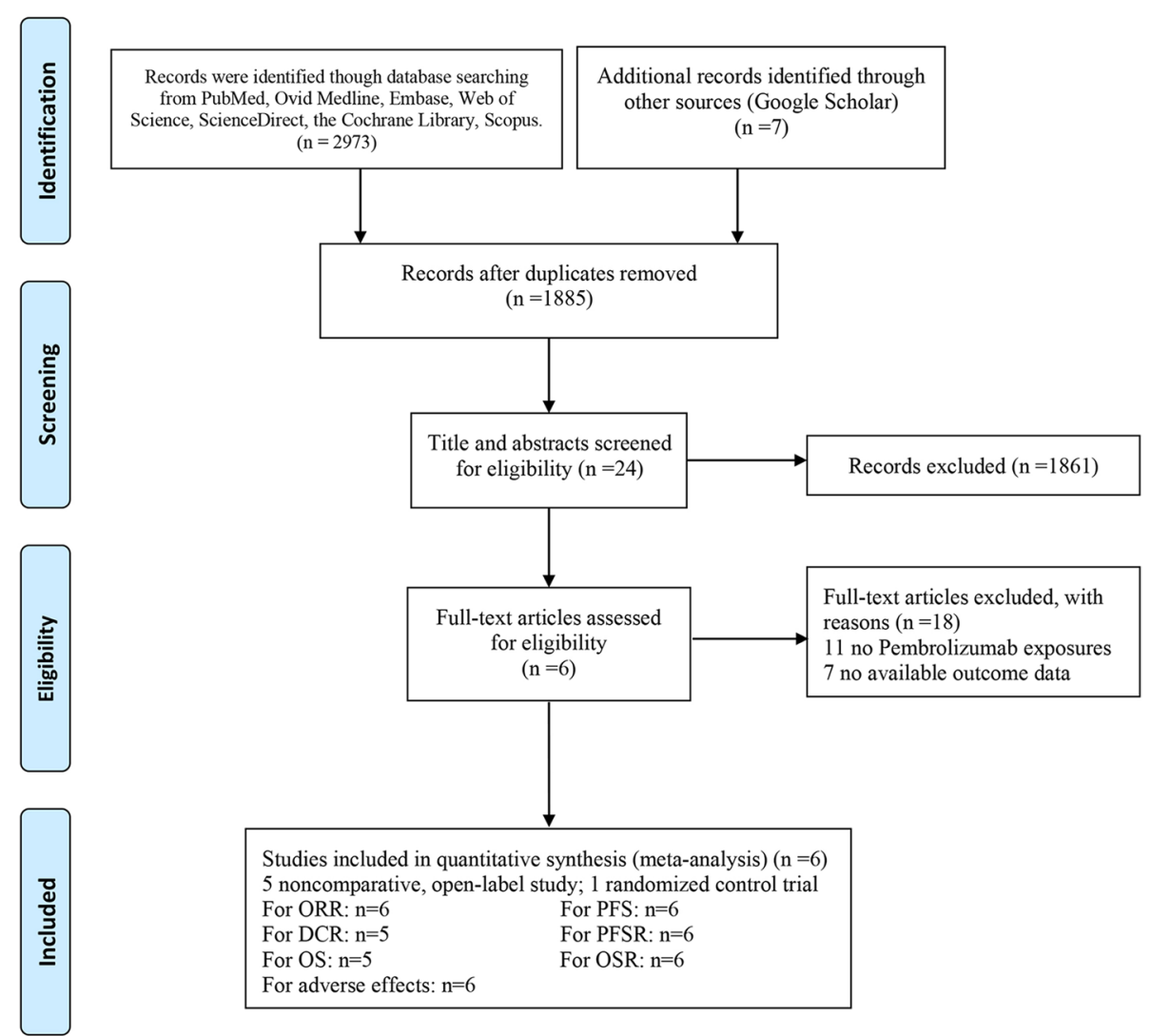

Fig. 1 Flow chart of study selection

(95\% CI, 2.2-6.1, $I^{2}=92.0 \%$ ) (Table S5, Fig. 2B), with the progression-free survival rate at 3 months (PFSR-3m), PFSR-6m, PFSR-9m, PFSR-12m, and PFSR-15m being 75.4\% (95\% CI, 62.3-88.5\%), 41.60\% (95\% CI, 24.3-59.0\%), 29.5\% (95\% CI, 13.6-44.9\%), 23.9\% (95\% CI, 10.2-37.6\%), and $10.0 \%$ (95\% CI, 6.8-13.3\%) (Fig. 3), respectively.

In some studies reporting drug response, the pooled ORR [12-17] was $38.80 \%$ (95\% CI, 11.9-65.67\%, $I^{2}=$ 97.2\%) (Table S6, Fig. 2C); the pooled DCR [11, 12, 14-16] was $69.30 \%$ (95\% CI, 51.6-87.0\%) (Table S7), the pooled CR was $2.20 \%$ (95\% CI, 0.8-3.7\%), the pooled PR was $34.70 \%$ (95\% CI, 7.8-61.5\%), and the pooled SD was 20.90\% (95\% CI, 9.1-32.6\%) (Table S8).

\section{Toxicity}

We summarized the total AEs in all of the included studies (Table 3). The most common AEs were neutropenia (90.16\%), dysphagia (41.96\%), platelet count decrease (34.87\%), anemia (53.21\%), esophagitis (32.89\%), alopecia $(32.89 \%)$, fatigue $(32.7 \%)$, nausea $(32.51 \%)$, decreased appetite (29.39\%), and decreased white blood cell count (26.3\%).

Some studies evaluated the grade 3-4 AEs\% [12-17] involving 391 patients. We gathered all grade 3-4 AEs in the included studies (Table S9); the pooled grade 3-4 AE rate was $20.88 \%$ (95\% CI, $1.22-54.85 \%, I^{2}=98.6 \%$ ) (Table S10, Fig. 4A). The severe AEs included neutropenia $(36.68 \%)$, anemia $(14.19 \%)$, pneumonitis $(6.57 \%)$, acute coronary syndrome (4.44\%), colitis/intestinal ischemia (4.17\%), and respiratory failure (2.5\%).

Some studies reported discontinuation treatment rate [12-17] involving 396 patients; the pooled discontinuation treatment rate was $9.50 \%$ (95\% CI, 4.40-14.70\%; $I^{2}=$ $54.2 \%, P=0.068$ ) (Table S11, Fig. 4B).

\section{Subgroup analysis}

To confirm whether the efficacy of pembrolizumab plus chemotherapy varied among different subgroups, all subgroup effects for the OS, PFS, and ORR were calculated in every category of the relevant variables. The results of most subgroups were consistent with the overall results. 


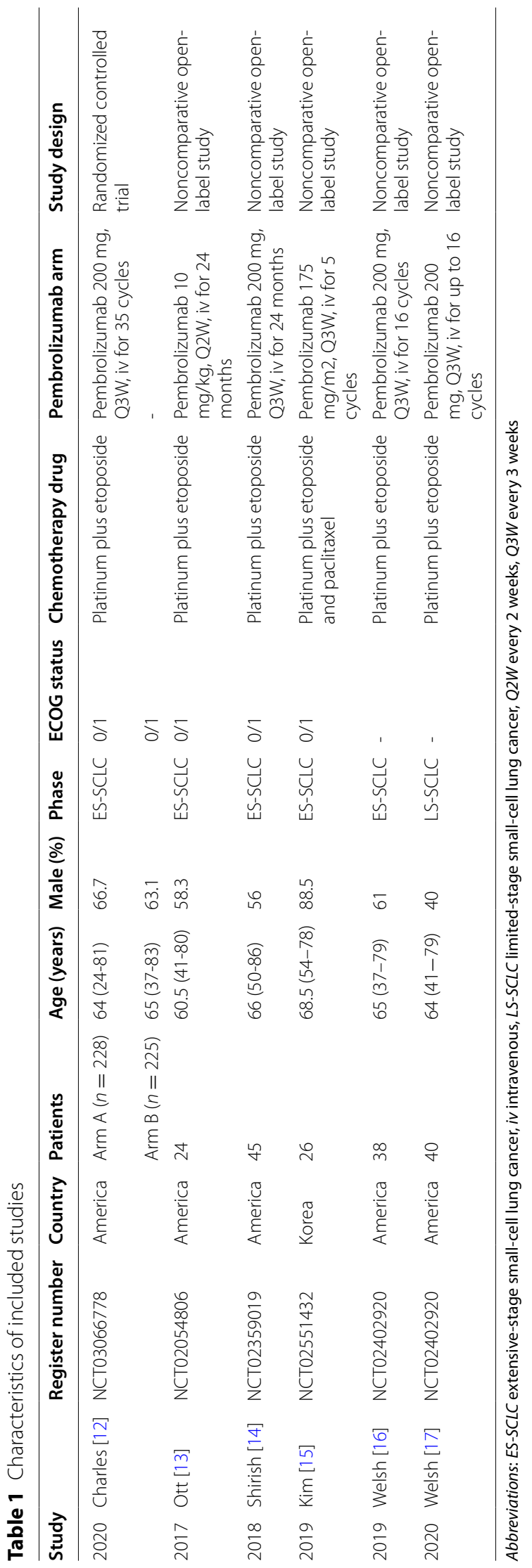




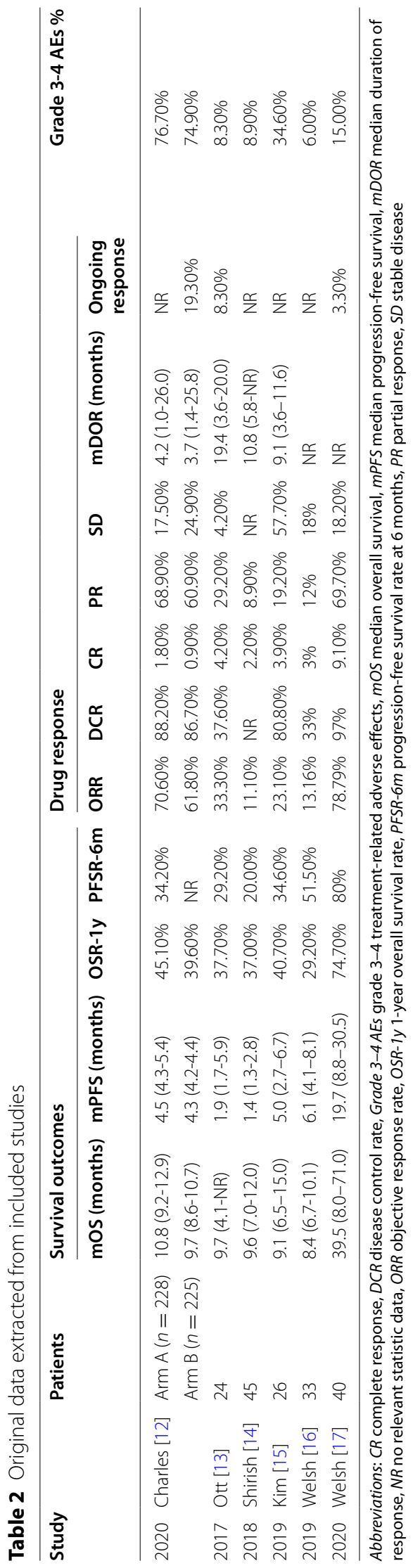


A

Study

ID

ES $(95 \%$ CI $) \quad$ Weight

$20170 t$

2019Kim

2019Welsh

2020Charles

2020Welsh

Overall $(\mathrm{I}-$-squared $=43.1 \%, \mathrm{p}=0.134)$

NOTE: Weights are from random effects analysis

$-71$

B

Study

$\%$

ID

ES $(95 \% \mathrm{CI}) \quad$ Weight

\section{Ott}

2018Shirish

2019Kim

2019Welsh

2020Charles

2020Welsh

Overall $(\mathrm{I}-\mathrm{squared}=92.0 \%, \mathrm{p}=0.000)$

NOTE: Weights are from random effects analysis

$\mathrm{C}$

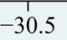

$-30.5$

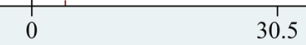

$9.6(7.0,12.0) \quad 23.55$

$9.1(6.5,15.0) \quad 11.60$

$8.4(6.7,10.1) \quad 33.32$

$10.8(9.2,12.9) \quad 31.26$

$39.5(8.0,71.0) \quad 0.27$

$9.6(8.0,11.2) \quad 100.00$

1

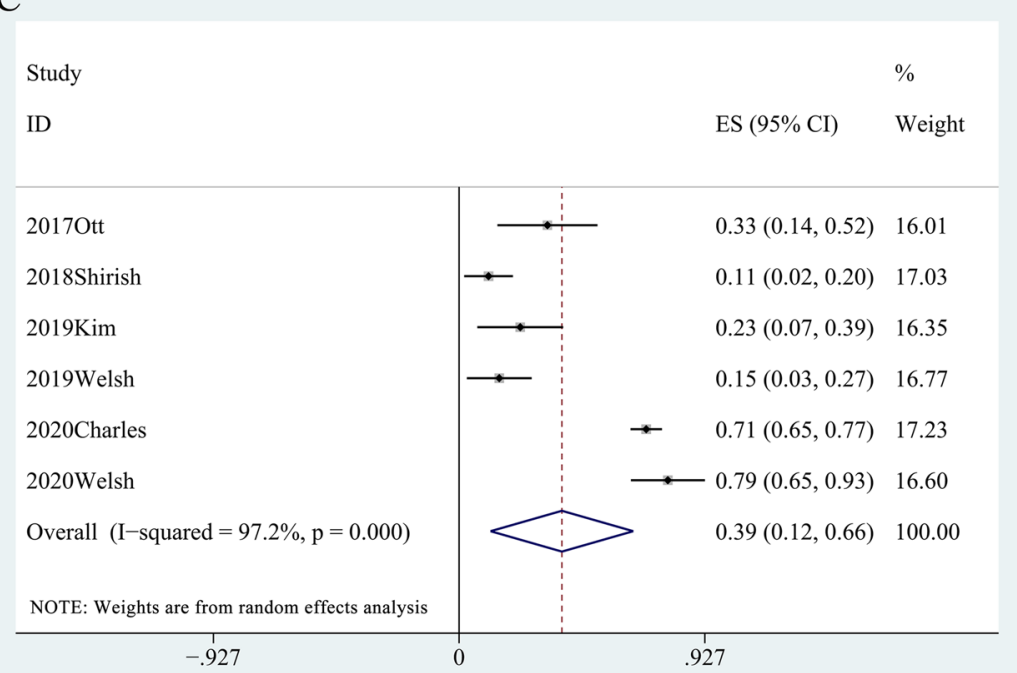

Fig. 2 Forest plot of $m O S$ A, $m P F S$ B, and ORR C 

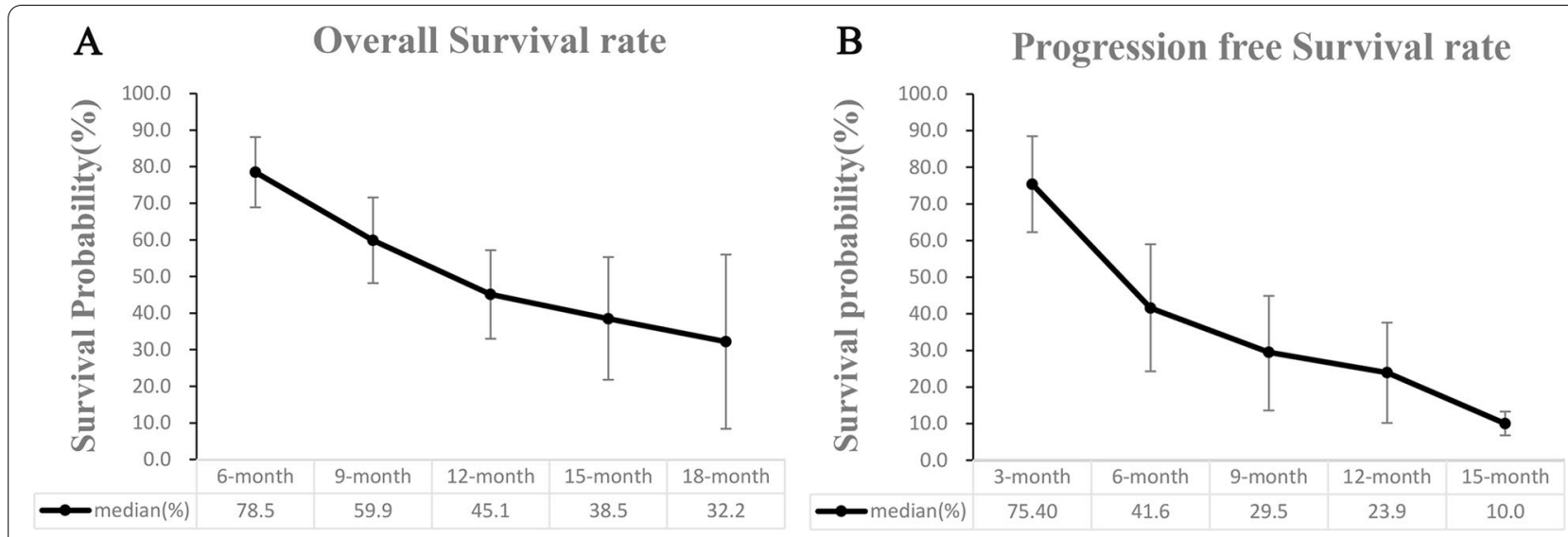

Fig. 3 Tendency chart of OS rate (6-18 months, A) and PFS rate (3-15 months, B)

Subgroup analysis indicated the antitumor activity of the combination of pembrolizumab and chemotherapy (Q2W, $200 \mathrm{mg}$ for 16 cycles) was superior to chemotherapy alone in mOS, mPFS, and ORR. The patients with a median age $\leq 65$ years old and with LS-SCLC also showed a better response. No transparent influence in study design, chemotherapy drug, or region was observed. Table 4 lists the results of the subgroup analyses.

\section{Comparison with chemotherapy alone}

Only one RCT compared pembrolizumab plus chemotherapy (etoposide and platinum [EP]) and chemotherapy alone (EP) [12]. The results showed that pembrolizumab plus chemotherapy remarkably advanced PFS (hazard ratio $[\mathrm{HR}]=0.75 ; 95 \% \mathrm{CI}, 0.61-0.91 ; P=0.0023)$ and prolonged OS $(\mathrm{HR}=0.80 ; 95 \% \mathrm{CI}=0.64-0.98 ; P=$ $0.0164)$. The ORR was $70.6 \%$ in the pembrolizumab plus chemotherapy group and $61.8 \%$ in the chemotherapy alone group, and the grade 3-4 AEs were 76.7 and $74.9 \%$, respectively.

\section{Sensitivity analysis}

Because high heterogeneity was found in mPFS, ORR, and grade 3-4 AEs, we evaluated the impact of every study on the pooled results to demonstrate stability and sensitivity. The results revealed that the outcomes of mOS (Figure S1A), mPFS (Figure S1B), ORR (Figure $\mathrm{S} 1 \mathrm{C}$ ), and grade 3-4 AEs (Figure S1D) were reliable and stable.

\section{Publication bias}

The publication bias of the pooled mOS (Egger's test: $P$ $=0.388$; Begg's test: $P=0.806$; Figure S2A) and pooled mPFS (Egger's test: $P=0.925$; Begg's test: $P=0.851$; Figure S2B) were examined with Egger's and Begg's regression tests. The results did not reveal any publication bias in mOS and mPFS.

\section{Discussion}

SCLC is highly aggressive and associated with poor survival outcomes, and it lacks optional and effective treatment arms. Although SCLC is sensitive to conventional first-line chemotherapy drugs, most patients relapse [29]. The efficacy of pembrolizumab plus conventional chemotherapy remains controversial. This meta-analysis is the first to analyze the efficacy and risks of the combination of pembrolizumab and conventional chemotherapy in SCLC patients. Our results showed that pembrolizumab is a promising immunotherapy drug for combination with conventional chemotherapy drugs.

In the analysis of survival data, we found that the pooled mOS, pooled mPFS, and pooled ORR were 9.6 months with $95 \%$ CI, 8.0-11.2, 4.2 months with 95\% CI, 2.2-6.1, and $38.8 \%$ with $95 \%$ CI, 11.9-65.67\%, respectively. Charles et al. [12] compared pembrolizumab plus chemotherapy with chemotherapy alone and demonstrated that the combination of pembrolizumab plus chemotherapy can improve mOS (HR, 0.80; 95\% CI, 0.64-0.98; $P=0.0164$ ) and mPFS (HR, 0.75; 95\% CI, 0.61-0.91; $P=$ 0.0023) without unexpected toxicities. Baize et al. [29] evaluated the efficacy of first-line chemotherapy alone in 162 patients, and their results showed that the mOS and mPFS were 7.5 months and 4.7 months, respectively, and the ORR was $49 \%$ in SCLC. Chung et al. [8] evaluated the efficacy of pembrolizumab alone in 107 SCLC patients, and the results showed that the MPFS and mOS were 2.0 months and 9.1 months, respectively; the ORR was $18.7 \%$ in SCLC. As demonstrated by the above results, we propose that pembrolizumab combined with chemotherapy is superior to a single agent. Based on this finding, we conducted further analyses. Via the programmed cell 
Table 3 Total adverse effects in SCLC patients

\begin{tabular}{|c|c|c|c|}
\hline Adverse events & $\begin{array}{l}\text { Studies } \\
\text { involved }\end{array}$ & Event/total & $\%$ \\
\hline \multicolumn{4}{|l|}{ Hematological system AEs } \\
\hline Neutropenia & 2 & $229 / 254$ & 90.16 \\
\hline Anemia & 4 & $174 / 327$ & 53.21 \\
\hline Platelet count decrease & 2 & $91 / 261$ & 34.87 \\
\hline WBC count decrease & 4 & $91 / 346$ & 26.30 \\
\hline Febrile neutropenia & 2 & $7 / 66$ & 10.61 \\
\hline \multicolumn{4}{|l|}{ Digestive system AEs } \\
\hline Dysphagia & 2 & $30 / 73$ & 41.96 \\
\hline Esophagitis & 2 & $25 / 73$ & 32.89 \\
\hline Nausea & 5 & $118 / 363$ & 32.51 \\
\hline Constipation & 4 & $83 / 332$ & 25.00 \\
\hline Decreased appetite & 1 & $67 / 228$ & 29.39 \\
\hline Diarrhea & 5 & $76 / 363$ & 20.94 \\
\hline Vomiting & 2 & $40 / 254$ & 15.75 \\
\hline $\begin{array}{l}\text { Increased aspartate transaminase } \\
\text { level }\end{array}$ & 1 & $6 / 45$ & 13.33 \\
\hline Norexia & 3 & 13/99 & 13.13 \\
\hline Abdominal pain & 1 & $5 / 45$ & 11.11 \\
\hline Stomatitis & 1 & $1 / 26$ & 3.85 \\
\hline Duodenitis & 1 & $1 / 40$ & 2.50 \\
\hline Pancreatitis & 1 & $1 / 40$ & 2.50 \\
\hline \multicolumn{4}{|l|}{ Respiratory system AEs } \\
\hline Dyspnea & 4 & $76 / 346$ & 21.97 \\
\hline Pneumonia & 3 & $53 / 294$ & 18.03 \\
\hline Lung infection & 1 & $5 / 40$ & 12.50 \\
\hline Respiratory failure & 1 & $1 / 40$ & 2.50 \\
\hline \multicolumn{4}{|l|}{ Skin AEs } \\
\hline Rash & 6 & $51 / 396$ & 12.88 \\
\hline Pruritus & 5 & $46 / 372$ & 12.37 \\
\hline Dry skin & 1 & $2 / 24$ & 8.33 \\
\hline Excessive tearing & 1 & $2 / 24$ & 8.33 \\
\hline Radiation dermatitis & 2 & $6 / 73$ & 8.22 \\
\hline \multicolumn{4}{|l|}{ Circulative system AEs } \\
\hline Peripheral edema & 2 & $22 / 268$ & 8.21 \\
\hline Pericarditis & 1 & $1 / 40$ & 2.50 \\
\hline Sinus tachycardia & 1 & $1 / 40$ & 2.50 \\
\hline Flushing & 1 & $1 / 40$ & 2.50 \\
\hline \multicolumn{4}{|l|}{ Nervous system AEs } \\
\hline Peripheral sensory neuropathy & 2 & $17 / 66$ & 25.76 \\
\hline Peripheral motor neuropathy & 1 & $3 / 26$ & 11.54 \\
\hline Neuropathy & 1 & $5 / 45$ & 11.11 \\
\hline \multicolumn{4}{|l|}{ Motor system AEs } \\
\hline Myalgia & 2 & $12 / 50$ & 24.00 \\
\hline Arthralgia & 2 & $10 / 64$ & 15.63 \\
\hline Back pain & 2 & $33 / 273$ & 12.09 \\
\hline Arthritis & 1 & $2 / 40$ & 5.00 \\
\hline Muscle weakness & 1 & $1 / 33$ & 3.03 \\
\hline Chest wall pain & 1 & $1 / 40$ & 2.50 \\
\hline
\end{tabular}

Table 3 (continued)

\begin{tabular}{|c|c|c|c|}
\hline Adverse events & $\begin{array}{l}\text { Studies } \\
\text { involved }\end{array}$ & Event/total & $\%$ \\
\hline \multicolumn{4}{|c|}{ Electrolyte disturbance AEs } \\
\hline Hyponatremia & 3 & $32 / 294$ & 10.88 \\
\hline Hypokalemia & 1 & $3 / 40$ & 7.50 \\
\hline \multicolumn{4}{|l|}{ Urological system AEs } \\
\hline Creatinine increased & 1 & $9 / 40$ & 22.50 \\
\hline Chronic kidney disease & 1 & $2 / 40$ & 5.00 \\
\hline \multicolumn{4}{|l|}{ Endocrine system AEs } \\
\hline Type I diabetes mellitus & 1 & $3 / 26$ & 11.54 \\
\hline Hypothyroidism & 2 & $24 / 254$ & 9.49 \\
\hline \multicolumn{4}{|l|}{ Systemic AEs } \\
\hline Fatigue & 5 & $121 / 370$ & 32.70 \\
\hline Asthenia & 3 & $58 / 278$ & 20.86 \\
\hline Pyrexia & 1 & $35 / 228$ & 15.35 \\
\hline Generalized weakness & 1 & $5 / 45$ & 11.11 \\
\hline \multicolumn{4}{|l|}{ Others AEs } \\
\hline Alopecia & 1 & $75 / 228$ & 32.89 \\
\hline Cough & 4 & $71 / 346$ & 20.52 \\
\hline Dizziness & 3 & $48 / 229$ & 16.05 \\
\hline Noncardiac chest pain & 1 & $6 / 45$ & 13.33 \\
\hline Headache & 4 & $39 / 332$ & 11.75 \\
\hline Insomnia & 2 & $27 / 252$ & 10.71 \\
\hline Pain & 1 & $3 / 33$ & 9.09 \\
\hline Confusion & 1 & $1 / 40$ & 2.50 \\
\hline
\end{tabular}

Abbreviations: AEs adverse effects, SCLC small-cell lung cancer

death 1 (PD-1) signaling pathway, the release of negative regulators of immune activation (immune checkpoints) restricts antitumor responses and leads to unparalleled rates of persistent tumor responses in patients with multifarious cancers [30]. Pembrolizumab is a highly efficient monoclonal antibody with high selectivity that can directly handicap the correlation of PD-1 and PD-L1 or PD-L2 [31, 32]. However, the expression of PD-L1 and PD-L2 in SCLC is lower than that in non-smallcell lung cancer (NSCLC) [33]. Therefore, monotherapy with pembrolizumab has certain limitations [34]. Second, the emergence of drug resistance and decreased chemotherapy efficacy remains a clinical challenge [7]. Third, Melosky hypothesized that chemotherapy can be a potential regulator for PD-L1 expression, which may promote a tumor cell's response to pembrolizumab [35]. A promising comprehensive treatment strategy for SCLC would be a significant advancement [36]. We propose that pembrolizumab plus chemotherapy may have better antitumor efficacy to prolong OS and PFS in SCLC than chemotherapy alone or pembrolizumab alone.

In subgroup analyses, age and SCLC phase were the main factors influencing the efficacy of pembrolizumab 


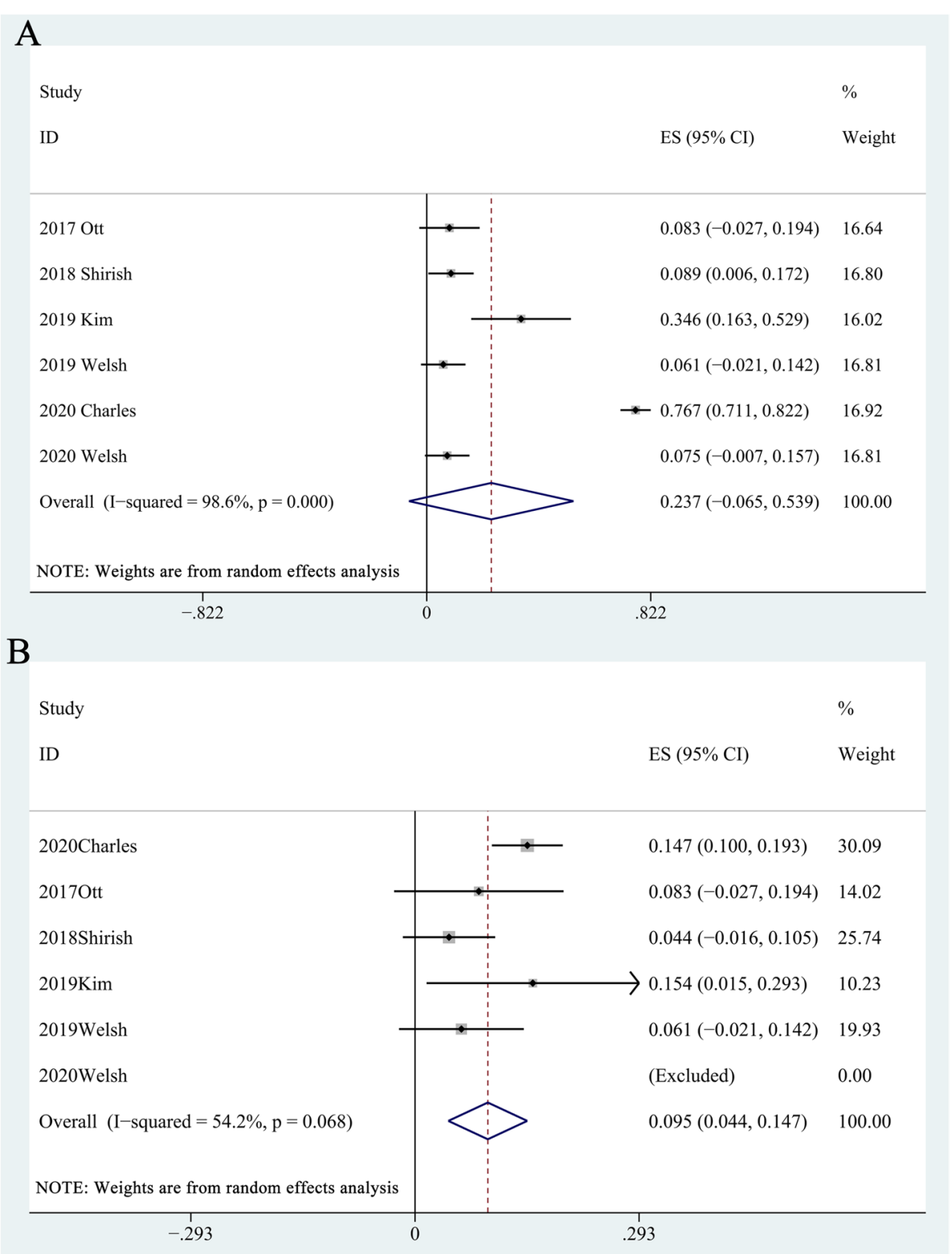

Fig. 4 Forest plots of grade 3-4 adverse effects rate $(\mathbf{A})$ and discontinuation treatment rate (B)

plus chemotherapy. Patients with a median age $<65$ years old had better results than the subgroup of patients $>65$ years old. Several factors may account for this finding. First, a higher tumor mutation burden (TMB) was used for prediction of better durable clinical benefit (DCB) of immune checkpoint inhibitors (ICIs) in younger patients [37]. The poorer response of older patients to ICIs may be related to immunosenescence characterized by decreased $\mathrm{T}$ cell proliferation, which is considered an age-related change in the immunity of the host, suggesting that patients with increased age had a worse response to ICI therapy that may influence the efficacy of ICIs in elderly people with a high prevalence of malignancies [38]. Finally, treatment-related AEs occur less often in younger patients than in older patients. Patients with LS-SCLC have better organ function reserve with a more sensitive drug response [17] and therefore a longer survival. As a PD1/PD-L1 inhibitor, PD1 expression level may be also the key factor of efficacy of Pembrolizumab plus chemotherapy [39]; besides, smoking may also influence the response to pembrolizumab [40]. Unfortunately, we have not obtained enough data to analyze the impact 


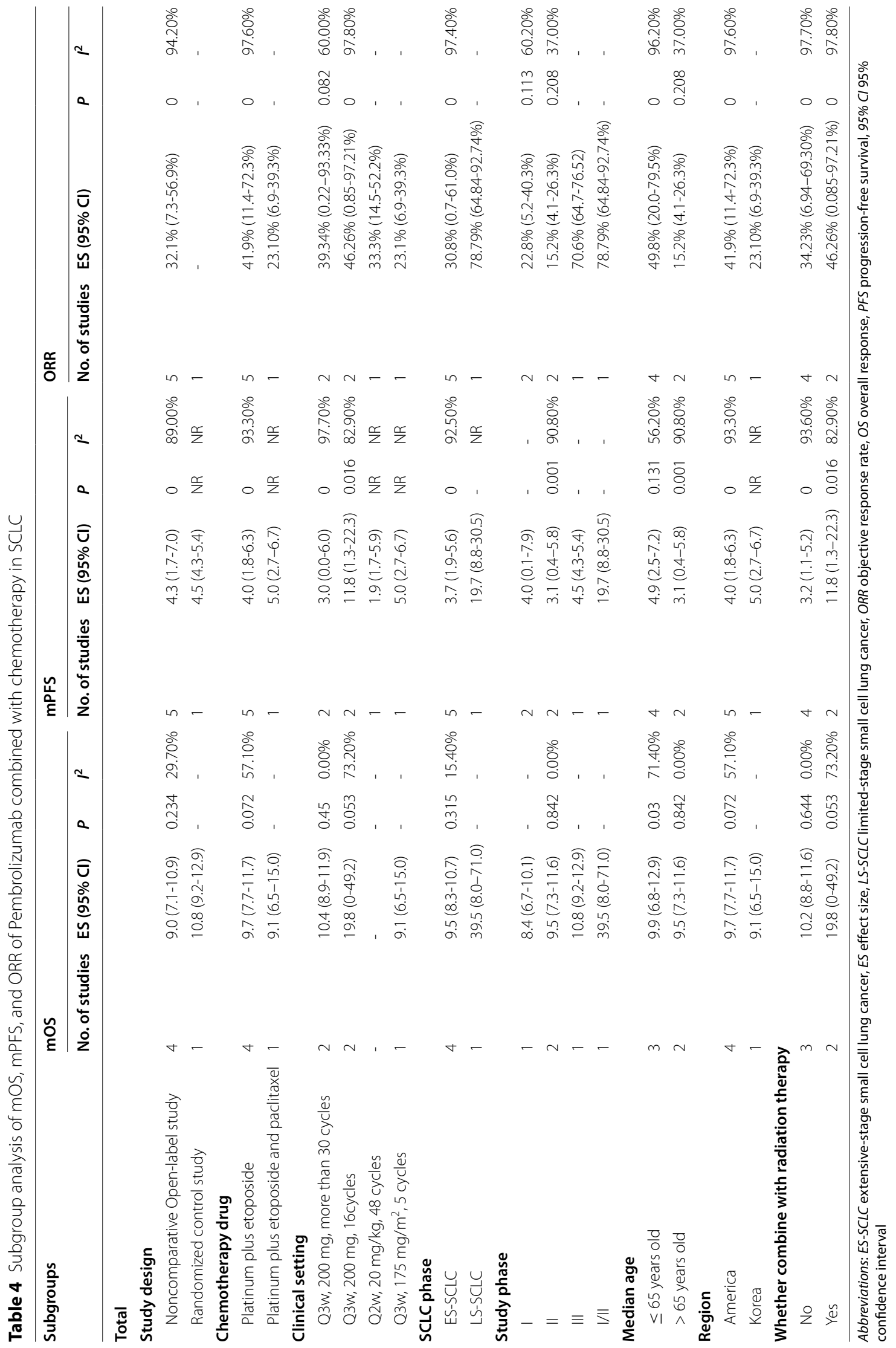


of these two factors. We will continue to follow up this research in the future.

We also analyzed the AEs of pembrolizumab combined with chemotherapy, including all grade AEs and grade 3-4 AEs. The most common AEs were neutropenia (90.16\%), anemia (53.21\%), dysphagia (41.96\%), platelet count decrease (34.87\%), esophagitis (32.89\%), alopecia (32.89\%), fatigue (32.7\%), nausea (32.51\%), decreased appetite $(29.39 \%)$, and white blood cell count decrease (26.3\%), which were manageable and similar to those reported in other solid tumor types, including SCLC [41]. Hematological system relevant AEs (including neutropenia, anemia, and platelet count decrease) and checkpoint inhibitor pneumonia (CIP) should be given attention. First- or second-line chemotherapy that damages bone marrow is the most common cause of leukopenia (chemotherapy-induced myelosuppression, CIM), which is more likely to occur in elderly patients and can be associated with decreased survival and tumor response [42], so control of CIM in the treatment of SCLC is crucial. CIP is a severe immune-related adverse event (irAE) that can complicate ICI therapy and is potentially life-threatening [40]. No definitive evidence has discerned the exact mechanism of CIP, but current evidence demonstrated that CIP is a lung disease with inflammatory infiltration due to autoantibody-mediated immunological processes with upregulated $\mathrm{T}$ cell activities, cytokine production levels, and amplification of complement [43]. CIP should be given more attention because of its considerable harm [44], although the incidence is low (18.03\% in our study). In conclusion, pembrolizumab combined with chemotherapy is relatively safe in SCLC patients, but hematological system relevant AEs and CIP need to be treated carefully.

Several limitations should be considered when interpreting our results. First, although we included almost all of the up-to-date studies in this emerging field, only 6 studies with a relatively small number of eligible participants were included in our meta-analysis. Second, all the included documents were written in English, which may lead to language bias. Third, only one study analyzed the results of pembrolizumab plus chemotherapy compared with chemotherapy alone. Because almost all the included studies lacked control or therapy groups without available control group data, we subjectively assessed the efficacy and risk of selection bias without an exact direct statistical conclusion. We lacked sufficient data on PD-1/PD-L1 expression to analyze its correlation with the efficacy and risk of pembrolizumab. Fourth, although sensitive analysis demonstrated our outcomes of pooled mPFS, ORR, and grade 3-4 AEs were reliable and stable and subgroup analysis demonstrated there was no obvious impact on total pooled outcomes, the LS-SCLC, and second-line chemotherapy in our included studies may cause possible impact in outcome. Finally, the inhomogeneity between the level of participants and the level of the trial led to significant heterogeneity.

\section{Conclusion}

In summary, pembrolizumab with conventional chemotherapy is a beneficial therapeutic schedule with assessable PFS, OS, ORR, and grade 3-4 AEs. Subgroup analyses indicated that patient age $(<65$ years old $)$ and SCLC phase (LS-SCLC) are the main factors affecting efficacy of pembrolizumab plus chemotherapy, but treatment-related hematological system AEs and CIP should be given attention during treatment. More high-quality RCTs with large samples are needed to further validate our conclusions.

\section{Abbreviations}

AEs: Adverse effects; Cl: Confidence interval; CR: Complete response; CIP: Checkpoint inhibitor pneumonia; CIM: Chemotherapy-induced myelosuppression; DCB: Durable clinical benefit; DCR: Disease control rate; ES: Effects sizes; EP: Etoposide and platinum; ECOG status: Eastern Cooperative Oncology Group performance status; FDA: US Food and Drug Administration; HR: Hazard ratio; irAEs: Immune-related adverse event; ICl: Immune checkpoint inhibitors; LI: Lower interval; mOS: Median overall survival; mPFS: Median progression free survival; mDOR: Median duration of response; OSR-12mo: Overall survival rate at 12 months; OS: Overall survival; ORR: Objective response rate; PD-L1/ PD-1: Programmed death ligand 1; PFS: Progression-free survival; PFSR-3m: Progression-free survival rate at 3 months; PR: Partial response; PRISMA: Preferred Reporting Items for Systematic Review and Meta-Analysis; RCT: Randomized control trial; TBM: Tumor mutation burden; SCLC: Small-cell lung cancer; SD: Stable disease; UL: Upper interval.

\section{Supplementary Information}

The online version contains supplementary material available at https://doi. org/10.1186/s12957-021-02410-3.

Additional file 1: Figure $\mathbf{S}$ 1. Sensitivity analysis of $\mathrm{mOS}(\mathrm{A}), \mathrm{mPFS}(\mathrm{B})$, ORR (C) and grade 3-4 adverse effects (D).

Additional file 2: Figure S2. Publication bias of mOS (A) and mPFS (B). Additional file 3: Table S1. PRISMA 2009 Checklist.

Additional file 4: Table S2. Search strategy.

Additional file 5: Table S3. GRADE Quality assessment by therapeutic strategy and study design for the outcomes of survival, and adverse events.

Additional file 6: Table S4. Pooled median overall survival in SCLC patients.

Additional file 7: Table S5. Pooled median progression-free survival in SCLC patients.

Additional file 8: Table S6. Pooled objective response rate in SCLC patients.

Additional file 9: Table S7. Pooled disease control rate in SCLC patients. Additional file 10: Table S8. Confirmed efficacy results (investigatorassessed) in the total population.

Additional file 11: Table S9. Pooled discontinuation treatment rate in SCLC patients for included studies. 
Additional file 12: Table S10. Pooled grade 3-4 adverse effects rate in SCLC patients.

Additional file 13: Table S11. Grade 3-4 adverse effects in included studies.

\section{Acknowledgements}

The authors thank professor Jianjun Xu, MD (Department of Thoracic Surgery, The Second Affiliated Hospital of Nanchang University) for his statistical advice.

\section{Authors' contributions}

Qiangyun Liu had full access to all of the data in the manuscript and takes responsibility for the integrity of the data and the accuracy of the data analysis. Concept and design: All authors. Acquisition, analysis, or interpretation of data: All authors. Drafting of the manuscript: Qiangyun Liu, Shuqiang Zhu, and Wenxiong Zhang. Critical revision of the manuscript for important intellectual content: Qiangyun Liu, Yixuan Zhang, Miaowen Liu, Ruoxin Xu, Fengming Yi, and Yiping Wei. Statistical analysis: Qiangyun Liu, Yixuan Zhang, Miaowen Liu, and Ruoxin Xu. Supervision: Qiangyun Liu, Shuqiang Zhu, and Wenxiong Zhang. The authors read and approved the final manuscript.

\section{Funding}

This study was supported by National Natural Science Foundation of China (NSFC), number of grants (81560345), Natural Science Foundation of Jiangxi Province (Grant number: 20181BAB215027). The funders had no role in the design and conduct of the study; collection, management, analysis, and interpretation of the data; preparation, review, or approval of the manuscript: and decision to submit the manuscript for publication.

\section{Availability of data and materials}

The data sets used and/or analyzed during the current study are available from the corresponding author on reasonable request.

\section{Declarations}

\section{Ethics approval and consent to participate}

This article does not contain any studies with human participants or animals performed by any of the authors.

\section{Consent for publication}

Not applicable.

\section{Competing interests}

The authors declare that they have no competing interests.

\section{Author details}

'Department of Thoracic Surgery, The Second Affiliated Hospital of Nanchang University, 1 Minde Road, Nanchang 330006, China. ${ }^{2}$ Jiangxi Medical College, Nanchang University, Nanchang 330006, China. ${ }^{3}$ Department of Oncology, The Second Affiliated Hospital of Nanchang University, 1 Minde Road, Nanchang 330006, China. ${ }^{4}$ Department of Cardiovascular Surgery, The Second Affiliated Hospital of Nanchang University, Nanchang 330006, China.

Received: 10 August 2021 Accepted: 25 September 2021

Published online: 14 October 2021

\section{References}

1. Rebecca L, Siege, Miller KD. Cancer statistics, 2020. CA Cancer J Clin. 2020;70(1):7-23.

2. Saltos A, Shafique M, Chiappori A. Update on the biology, management, and treatment of small cell lung cancer (SCLC). Front Oncol. 2020;10:1074.

3. Esposito G, Palumbo G, Carillio G, Manzo A, Montanino A, Sforza $\checkmark$, et al. Immunotherapy in small cell lung cancer. Cancers (Basel). 2020;12(9):2522.

4. Fan Y, Zhao J, Wang QM, Huang DZ, Li XY, J Chen, et al. Camrelizumab plus apatinib in extensive-stage small-cell lung cancer
(PASSION): a multicenter, two-stage, phase 2 trial. J Thorac Oncol. 2020;S1556-0864(20)30806-6.

5. Nadia H, Gonçalo F, Meghan JM, Rafael M, Kong CY, Cronin KA, et al. The effect of advances in lung-cancer treatment on population mortality. N Engl J Med. 2020;383(7):640-9.

6. Ready N, Farago AF, de Braud F, Atmaca A, Hellmann MD, Schneider JG, et al. Third-line nivolumab monotherapy in recurrent SCLC: CheckMate 032. J Thorac Oncol. 2019;14(2):237-44.

7. Chen P, Kuang P, Wang L, Li W, Chen B, Yu L, et al. Mechanisms of drugsresistance in small cell lung cancer: DNA-related, RNA-related, apoptosisrelated, drug accumulation and metabolism procedure. Transl Lung Cancer Res. 2020;9(3):768-86.

8. Chung HC, Lopez-Martin JA, Kao SCH, Miller WH, Ros W, Gao B. Phase 2 study of pembrolizumab in advanced small-cell lung cancer (SCLC): KEYNOTE-158. J Clin Oncol. 2018;15(36):8506.

9. Gadgeel S, Abreu DR, Speranza G, Esteban E, Felip E, Dómine M, et al. Updated analysis from KEYNOTE-189: pembrolizumab or placebo plus pemetrexed and platinum for previously untreated metastatic nonsquamous non-small-cell lung cancer. J Clin Oncol. 2020;38(14):1505-17.

10. Yang S, Zhang Z, Wang QM. Emerging therapies for small cell lung cancer. J Hematol Oncol. 2019;12(1):47.

11. Stefan Z, Solange P. Delineating the role of PD-1/PD-L1 blockade in advanced SCLC. J Thorac Oncol. 2018;13(9):1242-4.

12. Rudin CM, Awad MM, Navarro A, Gottfried M, Peters S, Csőszi T, et al. Pembrolizumab or placebo plus etoposide and platinum as first-line therapy for extensive-stage small-cell lung cancer: randomized, double-blind, phase III KEYNOTE-604 study. J Clin Oncol. 2020;38(21):2369-79.

13. Ott PA, Elena E, Sandrine H, Dong WK, Anne M, Sanatan S, et al. Pembrolizumab in patients with extensive-stage small-cell lung cancer: results from the phase Ib KEYNOTE-028 study. J Clin Oncol. 2017;35(34):3823-9.

14. Gadgeel SM, Pennell NA, Fidler MJ, Halmos B, Bonomi P, Stevenson J, et al. Phase II study of maintenance pembrolizumab in patients with extensivestage small cell lung cancer (SCLC). J Thorac Oncol. 2018;13(9):1393-9.

15. Kim Y-J, Keam B, Ock C-Y, Song S, Kim M, Kim SH, et al. A phase II study of pembrolizumab and paclitaxel in patients with relapsed or refractory small-cell lung cancer. Lung Cancer. 2019;136:122-8.

16. Welsh JW, Heymach JV, Chen D, Verma V, Cushman TR, Hess KR, et al. Phase I trial of pembrolizumab and radiation therapy after induction chemotherapy for extensive-stage small cell lung cancer. J Thorac Oncol. 2019;15(2):266-73.

17. Welsh JW, Heymach JV, Guo C, Menon H, Klein K, Cushman TR, et al. Phase $1 / 2$ trial of pembrolizumab and concurrent chemoradiation therapy for limited-stage SCLC. J Thorac Oncol. 2020;S1556-0864(20):30713-9.

18. Hellen PJ, Gralla RJ, Kris MG, Potanovich LM. Quality of life assessment in individuals with lung cancer: testing the Lung Cancer Symptom Scale (LCSS). Eur J Cancer. 1993;29A(Suppl 1):S51-8.

19. Higgins JP, Altman DG, Gøtzsche PC, Jüni P, Moher D, Oxman AD, et al. The Cochrane collaboration's tool for assessing risk of bias in randomised trials. BMJ. 2011;343:d5928.

20. Guyatt GH, Oxman AD, Schünemann HJ, Tugwell P, Knottnerus A. GRADE guidelines: a new series of articles in the Journal of Clinical Epidemiology. J Clin Epidemiol. 2011;64(4):380-2.

21. Tian XW, Browning BL, Browning SR. Estimating the genome-wide mutation rate with three-way identity by descent. Am J Hum Genet. 2019;105(5):883-93.

22. Oxnard GR, Wilcox KH, Gonen M, Polotsky M, Hirsch BR, Schwartz $\mathrm{LH}$. Response rate as a regulatory end point in single-arm studies of advanced solid tumors. JAMA Oncol. 2016;2(6):772-9.

23. Syed AA, Rafe MJ, Huma Q, Sten HV. Hepatitis B and hepatitis C in Pakistan: prevalence and risk factors. Int J Infect Dis. 2009;13(1):9-19.

24. Sandroni C, D'Arrigo S, Callaway CW, Cariou A, Dragancea I, Taccone FS, et al. The rate of brain death and organ donation in patients resuscitated from cardiac arrest: a systematic review and meta-analysis. Intensive Care Med. 2016:42(11):1661-71.

25. Hozo SP, Djulbegovic B, Hozo I. Estimating the mean and variance from the median, range, and the size of a sample. BMC Med Res Methodol. 2005;5:13.

26. Higgins JP, Thompson SG, Deeks JJ, Altman DG. Measuring inconsistency in meta-analyses. BMJ. 2003;327(7414):557-60.

27. Spittal MJ, Pirkis J, Gurrin LC. Meta-analysis of incidence rate data in the presence of zero events. BMC Med Res Methodol. 2015;15:42. 
28. Langhorne P. Bias in meta-analysis detected by a simple, graphical test. Prospectively identified trials could be used for comparison with metaanalyses. BMJ. 1998;316(7129):471.

29. Baize N, Monnet I, Greillier L, Geier M, Lena H, Janicot H, et al. Carboplatin plus etoposide versus topotecan as second-line treatment for patients with sensitive relapsed small-cell lung cancer: an open-label, multicenter, randomized, phase 3 trial. Lancet Oncol. 2020:21(9):1224-33.

30. Ribas A, Wolchok JD. Cancer immunotherapy using checkpoint blockade. Science. 2018;359(6832):1350-5.

31. Zhao L, Ma B, Yang Y, Li T, Han L, Gao Q. Chemotherapy reverses anti-PD-1 resistance in one patient with advanced non-small lung cell cancer. Front Oncol. 2020;10:507.

32. lams WT, Porter J, Horn L. Immunotherapeutic approaches for small-cell lung cancer. Nat Rev Clin Oncol. 2020;17(5):300-12.

33. Lantuejoul S, Sound-Tsao M, Cooper WA, Girard N, Hirsch FR, Roden AC, et al. PD-L1 testing for lung cancer in 2019: perspective from the IASLC pathology committee. J Thorac Oncol. 2020;15(4):499-519.

34. Antonia SJ, López-Martin JA, Bendell J, Ott PA, Taylor M, Eder JP, et al. Nivolumab alone and nivolumab plus ipilimumab in recurrent small-cell lung cancer (CheckMate 032): a multicenter, open-label, phase 1/2 trial. Lancet Oncol. 2016;17(7):883-95.

35. Melosky B, Chu Q, Juergens RA, Leighl N, lonescu D, Tsao M-S, et al. Breaking the biomarker code: PD-L1 expression and checkpoint inhibition in advanced NSCLC. Cancer Treat Rev. 2018;65:65-77.

36. Sen T, Rodriguez BL, Chen L, Della Corte CM, Morikawa N, Fujimoto J, et al. Targeting DNA damage response promotes antitumor immunity through STING-mediated T-cell activation in small cell lung cancer. Cancer Discov. 2019;9(5):646-61.

37. Samstein RM, Lee C-H, Shoushtari AN, Hellmann MD, Shen R, Janjigian YY, et al. Tumor mutational load predicts survival after immunotherapy across multiple cancer types. Nat Genet. 2019;51(2):202-6.
38. Sun YM, Wang Y, Sun XX, Chen J, Gong ZP, Meng HY. Clinical efficacy of immune checkpoint inhibitors in older non-small-cell lung cancer patients: a meta-analysis. Front Oncol. 2020;10:558454.

39. Peng Z, Lin H, Zhou K, et al. Predictive value of pretreatment PD-L1 expression in EGFR-mutant non-small cell lung cancer: a meta-analysis. World J Surg Onc. 2021;19:145.

40. Mo J, Hu X, Gu L, et al. Smokers or non-smokers: who benefits more from immune checkpoint inhibitors in treatment of malignancies? An up-todate meta-analysis. World J Surg Oncol. 2020;18:15.

41. Chung HC, Piha-Paul SA, Lopez-Martin J, Schellens JHM, Kao S, Miller WH Jr, et al. Pembrolizumab after two or more lines of previous therapy in patients with recurrent or metastatic SCLC: results from the KEYNOTE-028 and KEYNOTE-158 studies. J Thorac Oncol. 2020;15(4):618-27.

42. Hart LL, Ferrarotto R, Andric ZG, Beck JT, Subramanian J, Radosavljevic DZ, et al. Myelopreservation with trilaciclib in patients receiving topotecan for small cell lung cancer: results from a randomized, double-blind, placebo-controlled phase II study. Adv Ther. 2021;38(1):350-65.

43. Li M, Spakowicz D, Zhao S, Patel SH, Johns A, Grogan M, et al. Brief report: inhaled corticosteroid use and the risk of checkpoint inhibitor pneumonitis in patients with advanced cancer. Cancer Immunol Immunother. 2020;69(11):2403-8.

44. Postow MA, Sidlow R, Hellmann MD. Immune-related adverse events associated with immune checkpoint blockade. N Engl J Med. 2018;378(2):158-68.

\section{Publisher's Note}

Springer Nature remains neutral with regard to jurisdictional claims in published maps and institutional affiliations.
Ready to submit your research? Choose BMC and benefit from:

- fast, convenient online submission

- thorough peer review by experienced researchers in your field

- rapid publication on acceptance

- support for research data, including large and complex data types

- gold Open Access which fosters wider collaboration and increased citations

- maximum visibility for your research: over $100 \mathrm{M}$ website views per year

At BMC, research is always in progress.

Learn more biomedcentral.com/submissions 